\title{
Posterior Reversible Encephalopathy Syndrome after Hypovolemic Shock Which Is Required Differential Diagnosis with Delirium in the Intensive Care Unit
}

\author{
Seo In Lee', Gyeong Seon $\mathrm{Choi}^{2}$, Jin Park', Young-Joo Lee', Kyung Sook Hong ${ }^{4}$ \\ Departments of ${ }^{1}$ Critical Care Medicine, ${ }^{2}$ Neurology and Critical Care Medicine, ${ }^{3}$ Anesthesiology and Critical Care Medicine, and ${ }^{4}$ Surgery and Critical Care \\ Medicine, Ewha Womans University College of Medicine, Seoul, Korea
}

Dear Editor:

Posterior reversible encephalopathy syndrome (PRES) represents various symptoms, such as unconsciousness, seizure, headache, or vision abnormalities, which are recovered in many cases after several days. PRES can be diagnosed relatively easily using neuroimaging, such as a magnetic resonance imaging (MRI), because of its characteristic lesions. PRES is reported with the several causes, for example, hypertensive crisis, immunosuppressive therapy, sepsis, eclampsia or preeclampsia, kidney injury, hemodialysis and blood transfusion [1,2]. In contrast to the name of PRES (which is "reversible"), symptoms and pathologic can be "irreversible" and delayed diagnosis can lead to secondary brain injury [3]. Thus, rapid diagnosis and appropriate treatment are very important.

Meanwhile, delirium is a very common psychiatric symptom seen in hospitals, especially in the intensive care unit (ICU), which is characterized by rapid changes in mental status with altered cognitive function [4]. Because of its high incidence [4], early symptoms of other neurological diseases such as PRES can be overlooked. Therefore, it is very important to differentiate the other neurological diseases so that diagnosis is not delayed.

Here we present a case of PRES after hypovolemic shock which was misdiagnosed as delirium. This brief report was approved by the Institutional Review Board (IRB No. EUMC 201803-042) and informed consent was waived by IRB.

A 41-year-old female patient was referred to the emergency department due to uterine bleeding after delivery in a local obstetric clinic. She had one vaginal delivery history before this pregnancy. She had no preeclampsia, eclampsia, or other notable events or history. The patient's demographic and clinical characteristics are shown in Table 1.

The patient underwent emergency vaginal delivery at the local obstetric clinic due to early placental abruption. Once post-partum bleeding was identified, she was transferred to the emergency department of our institution. After diagnosis of hypovolemic shock, central venous catheterization, massive transfusion, fluid resuscitation with crystalloid fluid, norepinephrine infusion and endotracheal intubation with ventilator care were performed (Table 1). Her blood pressure (BP) severely fluctuated; however, her vital signs were stabilized after two times of embolization (Figure 1A). Then norepinephrine was tapered before transferring to ICU. After transfer to the ICU, mechanical ventilation was applied and the endotracheal

\section{Letter to the Editor}

Received: August 17, 2018

Revised: September 11, 2018

Accepted: September 15, 2018

Corresponding author Kyung Sook Hong Department of Surgery and Critical Care Medicine, Ewha Womans University College of Medicine, 1071 Anyangcheon-ro, Yangcheon-gu, Seoul 07985, Korea

Tel: +82-2-2650-5366

Fax: +82-2-2650-5906

E-mail: hongks@ewha.ac.kr

Copyright (@) 2018 The Korean Society of Critical Care Medicine

This is an Open Access article distributed under the terms of Creative Attributions Non-Commercial License (http:// creativecommons.org/li-censes/by-nc/4.0/ which permits unrestricted noncommercial use, distribution, and reproduction in any medium, provided the original work is properly cited. 
Table 1. Demographic and clinical characteristics of patient

\begin{tabular}{|c|c|}
\hline Characteristics & Value \\
\hline Age (yr) & 41 \\
\hline Height (cm) & 162 \\
\hline Weight (kg) & 59 \\
\hline Body mass index $\left(\mathrm{kg} / \mathrm{m}^{2}\right)$ & 22.48 \\
\hline \multicolumn{2}{|l|}{ Menstruation } \\
\hline Interval (day) & 28 (Regular) \\
\hline Duration (day) & 5 \\
\hline Amount & Moderate \\
\hline Dysmenorrhea & - \\
\hline Parity & $2-0-1-2$ \\
\hline Initial fluid resuscitation & Crystalloid fluid 6,300 ml \\
\hline \multicolumn{2}{|l|}{ Transfusion (pint) } \\
\hline Packed red blood cell & 28 \\
\hline Fresh frozen plasma & 27 \\
\hline Plateletpheresis & 1 \\
\hline Cryoprecipitate & 5 \\
\hline \multicolumn{2}{|l|}{ Level of creatinine (mg/dl) } \\
\hline HD 1 & 1.04 \\
\hline HD 2 & 1.27 \\
\hline HD 3 & 1.12 \\
\hline HD 4 & 1.03 \\
\hline HD 5 & 0.91 \\
\hline HD 6 & 0.71 \\
\hline HD 7 & 0.61 \\
\hline HD 8 & 0.75 \\
\hline HD 9 & 0.91 \\
\hline HD 10 & 0.70 \\
\hline
\end{tabular}

HD: hospital day.

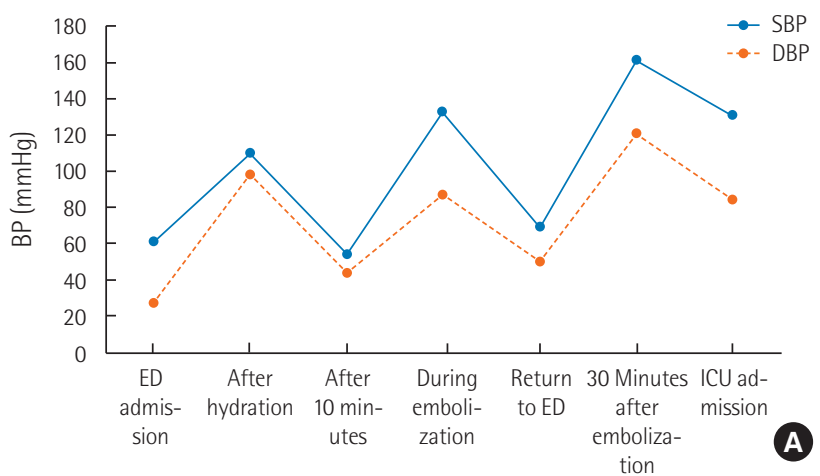

tube was removed on hospital day (HD) 5. Even she showed decreased urine output during ED admission (only $50 \mathrm{ml}$ for about 7 hours), urine output maintained after transferring to ICU. Also the level of creatinine elevated transiently on HD 2, and then it decreased without any procedures (Table 1).

Patient complained the headache on HD 6 and it subsided with pain control. On HD 7, the patient showed symptoms including shouting, swearing, acting out, aggressive behavior, hallucinations, self-talking, and trying to escape from bed and remove the catheters. There were no sensory or motor defects upon neurological examination. The Confusion Assessment Method for the ICU was performed and delirium was suspected. Therefore, haloperidol and lorazepam were administered intravenously as rapid tranquilizers and dexmedetomidine intravenous infusion was administered, and then it was switched to quetiapine fumarate with sips of water on HD 8, and agitation and acting out behavior were controlled.

However, hypoactive mood, aphasia, gazing at one-side, and abnormal movement of the lower lip were observed on HD 9. We suspected hypoactive-form delirium or over-sedation at first; however, MRI was performed to rule out pathological lesions due to atypical symptoms. A pathological white matter lesions was observed on MRI (Figure 2A). After reviewing the patient's vital chart retrospectively (Figure 1B), the patient was diagnosed with PRES. The symptoms mentioned above were considered as atypical manifestations of PRES. Gazing at one side could be the manifestation of visual disturbance and abnormal movement of the lower lip could be the kind of petit mal seizure.

We attempted to control the high $\mathrm{BP}$ with intravenous infusion of nicardipine hydrochloride immediately; symptoms

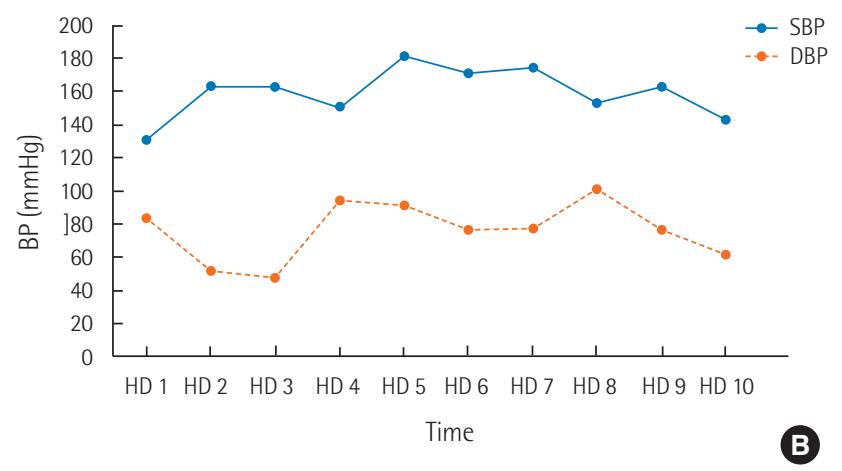

Figure 1. Changes of blood pressure (BP) over time. (A) BP fluctuation at the emergency department (ED). Patient showed the drastic fluctuation of BP. (B) BP changes during intensive care unit (ICU) admission. Patient showed relatively high BP from the hospital day (HD) 2 and posterior reversible encephalopathy syndrome related symptoms were identified from HD 6. SBP: systolic blood pressure; DBP: diastolic blood pressure. 

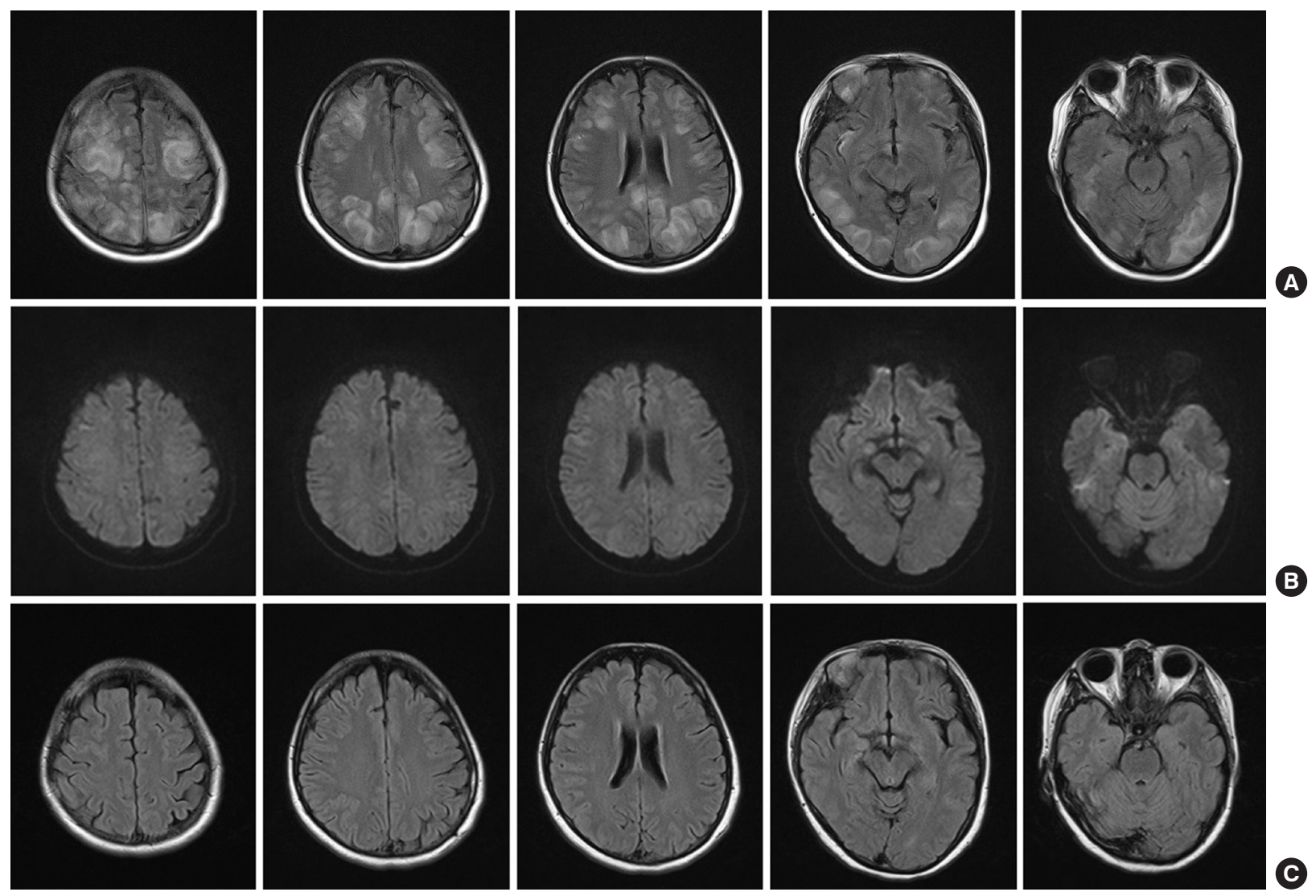

Figure 2. Magnetic resonance imaging (MRI) of patient with posterior reversible encephalopathy syndrome. (A) MRI on the time of diagnosis showed the typically hyperintense bilateral lesions. A pathological white matter lesion in the occipital, posterior parietal, and posterior temporal lobes was observed on T2-weighted and fluid-attenuated inversion recovery MRI. (B) Diffusion-weighted imaging of MRI on hospital day 9 showed no pathologic lesions. (C) All vasogenic edematous lesions were resolved on MRI followed-up after 77 days.

were recovered. The patient was transferred to the general ward on HD 12. The patient was discharged without any sequelae on HD 18, and repetitive MRI evaluations were performed through outpatient follow-up on the 77th day after PRES diagnosis (Figure 2C).

The retrospective review of the patient's medical records with the MRI findings suggested that no diagnosis other than PRES could be considered; however, when the first symptom occurred on HD 7, our medical team considered delirium as a priority. We diagnosed the patient with hyperactive-form delirium because of symptoms such as agitation, irritability, aggressive behavior, altered consciousness, and hallucinations, and we prescribed sedatives for delirium treatment.

The incidence of delirium in the ICU is reported to vary from $16 \%$ to $89 \%[4,5]$. Because cognitive impairment and attention deficits due to delirium may contributes to a negative prognosis [5], it is important to prevent or diagnose it as soon as possible and treat it appropriately. However, because of its high incidence, other neurologic diseases are often neglected.
When this patient's cognitive function and consciousness level changed during ICU admission due to hypovolemic shock, delirium was first suspected, and the patient was lightly sedated and the patient's circadian rhythm brought under control [5]. In particular, the risk of delirium is high in patients with mechanical ventilation therapy in the ICU [4]; therefore, we may have considered delirium without any other doubt. However, we found additional, uncommon symptoms (such as visual neglect, abnormal movement of the lower lip, etc.) and concluded that additional imaging tests were needed. After MRI examination, PRES was confirmed. In general, the diagnosis of PRES may be delayed due to its many causes and often atypical presentation. Delayed diagnosis of PRES may lead to permanent brain damage, and even to death in severe cases, so it is highly recommended to be concern in diagnosis [3].

In the present case, a patient without any previous history of hypertension showed severe hypovolemic shock at the time of admission. Then, the patient showed reactive hypertension. This level of high BP couldn't be concluded to be malignant or 
fatal; however, rapidly developing, fluctuating, or intermittent hypertension carries a particular risk for hypertensive encephalopathy [3]. Massive transfusion could be also the trigger of PRES in this patient [1,2]. In this case, 28 pints of packed red blood cells and 27 pints of fresh frozen plasma were administered at ED (Table 1) and there have been several reports related with transfusion-induced PRES. Also other vasogenic edematous encephalopathies, especially contrast-induced encephalopathy, should be considered. Contrast used during angiography or embolization could induce the vasogenic encephalopathy because of the disruption of blood-brain barrier [6]. However, the onset of encephalopathy was delayed from the usage of contrast and diffusion-weighted imaging in MRI (Figure 2B) showed no pathologic lesions in this patient, so we diagnosed as PRES.

Reviewing all findings in the present case, the causes of PRES in this patient are (1) BP fluctuation after hypovolemic shock, (2) an acute hypertensive event, (3) fluid overload, and (4) massive transfusion. When encountering patients in shock with various causes, proper fluid intake is a concern of many physicians, especially in the case of hypovolemic shock. In treating shock patients, physicians should be careful to ensure that the BP doesn't fluctuate and that fluid overload be avoided. Further, PRES is diagnosed quickly and acutely when symptoms are accompanied by seizure, but diagnoses are delayed if subacute progress is shown. In particular, intensivists who see critically ill patients should strive to prevent delirium and try to detect it early, but also be careful not to delay the diagnosis of other neurologic diseases due to an overbearing focus on the delirium.

\section{CONFLICT OF INTEREST}

No potential conflict of interest relevant to this article was reported.

\section{ORCID}

Seo In Lee

https://orcid.org/0000-0001-7036-6233

Gyeong Seon Choi

Jin Park

https://orcid.org/0000-0001-6616-7960

https://orcid.org/0000-0002-4597-6344

Young-Joo Lee

https://orcid.org/0000-0002-6512-3891

Kyung Sook Hong

https://orcid.org/0000-0002-8022-5693

\section{REFERENCES}

1. Lio CF, Lee YH, Chan HY, Yu CC, Peng NJ, Chan HP. Posterior reversible encephalopathy syndrome in a postpartum hemorrhagic woman without hypertension: a case report. Medicine (Baltimore) 2017;96:e6690.

2. Singh K, Gupta R, Kamal H, Silvestri NJ, Wolfe GI. Posterior reversible encephalopathy syndrome secondary to blood transfusion. J Clin Neurosci 2015;22:592-4.

3. Postma IR, Slager S, Kremer HP, de Groot JC, Zeeman GG. Long-term consequences of the posterior reversible encephalopathy syndrome in eclampsia and preeclampsia: a review of the obstetric and nonobstetric literature. Obstet Gynecol Surv 2014;69:287-300.

4. Reade MC, Finfer S. Sedation and delirium in the intensive care unit. N Engl J Med 2014;370:444-54.

5. Hong KS, Kim NR, Song SH, Hong G. Cycling of dexmedetomidine may prevent delirium after liver transplantation. Transplant Proc 2018;50:1080-2.

6. Shi F, Shen L, Shi Y, Shi L, Yang X, Jin Z, et al. Posterior reversible encephalopathy syndrome after postpartum hemorrhage and uterine artery embolization: a case report. Medicine (Baltimore) 2017;96:e8973. 